\title{
Bioelectrical impedance analysis; a new method to evaluate lymphoedema, fluid status, and tissue damage after gynaecological surgery - A systematic review
}

Madeleine Asklöf, Preben Kjölhede, Ninnie Borendal Wodlin and Lena Nilsson

The self-archived postprint version of this journal article is available at Linköping University Institutional Repository (DiVA):

http:// urn.kb.se/ resolve?urn=urn:nbn:se:liu:diva-152094

N.B.: When citing this work, cite the original publication.

Asklöf, M., Kjölhede, P., Borendal Wodlin, N., Nilsson, L., (2018), Bioelectrical impedance analysis; a new method to evaluate lymphoedema, fluid status, and tissue damage after gynaecological surgery - A systematic review, European J ournal of Obstetrics, Gynecology, and Reproductive Biology, 228, 111119. https:// doi.org/ 10.1016/j.ejogrb.2018.06.024

Original publication available at:

https:// doi.org/10.1016/j.ejogrb.2018.06.024

Copyright: Elsevier (12 months)

http://www.elsevier.com/

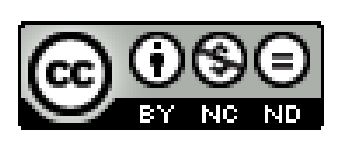




\section{A review article}

\section{entitled}

Bioelectrical impedance analysis; a new method to evaluate lymphoedema, fluid status, and tissue damage after gynaecological surgery - a systematic review.

by

${ }^{1}$ Madeleine Asklöf, BMedSci, ${ }^{1}$ Preben Kjølhede, MD, PhD, ${ }^{1}$ Ninnie Borendal Wodlin, MD PhD and ${ }^{2}$ Lena Nilsson, MD, PhD.

\section{Affiliations}

${ }^{1}$ Department of Obstetrics and Gynaecology, Department of Clinical and Experimental Medicine, Faculty of Medicine and Health Science, Linköping University, Linköping, Sweden

2 Department of Anesthesiology and Intensive Care, Department of Medical and Health Sciences, Linköping University, Linköping, Sweden.

Corresponding author:

Preben Kjølhede, MD, PhD

Department of Obstetrics and Gynaecology

University Hospital

S-581 85 Linköping

Sweden

Phone: +46101033187

Fax: +46 13148156

E-mail: Preben.Kjolhede@liu.se

Short running title Bioeletrical impedance and postoperative recovery

\section{Conflicts of interest}

None of the authors has conflicts of interest to declare. 


\section{Condensation}

This review reveals that there seems to be a wide range of promising applications for the BIA for predicting and eventually preventing postoperative complications in the gynaecological surgical patient. 


\section{Abstract}

The aim of this descriptive review is to summarise the current knowledge of non-invasive bioelectrical impedance analysis (BIA) used with gynaecological surgical patients in regard to postoperative development of lymphoedema and determination of perioperative fluid balance, and as a prognostic factor in cancer mortality and a predictor of postoperative complications.

The databases PubMed, MEDLINE, Scopus Web of Science, the Cochrane Library, and reference lists of selected articles were searched for relevant articles published during the period January 2008 to April 2018. Only papers published in English were retrieved. Thirty-seven articles were evaluated. Where gynaecological studies were lacking, studies with a study population from neighbouring clinical fields were used instead.

Studies on the clinical use of BIA with gynaecological surgical patients were divided into three categories: the postoperative development of lower limb lymphoedema $(n=7)$, perioperative hydration measuring $(\mathrm{n}=3)$, and the BIA parameter phase angle as a prognostic factor in cancer survival and as predictive for postoperative complications $(n=6)$. Of these 16 studies only three used a pure gynaecological study population. Three different methods of BIA were used in these articles: single frequency-BIA, multifrequency-BIA and bioimpedance spectroscopy. BIA was found to detect lymphoedema with a sensitivity of $73 \%$ and a specificity of $84 \%$. Studies indicated that BIA was able to detect lower limb lymphoedema at an early stage even before it became clinically detectable. During postoperative hydration measurements, an increase in extracellular fluid volume and extracellular fluid volume in relation to total body fluid volume, as well as a decrease in phase angle, were associated with higher frequencies of postoperative complications. Moreover, low values for the phase angle have been associated with increased mortality in cancer patients. However, the number of studies in this field was limited.

From our review, BIA seems to be a useful tool for use in the clinical setting of the gynaecological surgical patient. The theoretical approach of using bioelectrical impedance values to measure the fluid distribution in the body compartments offers wide opportunities in the clinical setting. 
However, so far, all studies have set up cut-off limits within the study population, and reference values for a general population need to be defined. There are also rather few studies on a gynaecological study population. Hence, there is a need for further studies within gynaecological surgery focusing on early detection of lower limb lymphoedema, perioperative fluid balance, and postoperative complications in order to establish the value of BIA in clinical praxis.

Keywords: Bioelectrical impedance analysis; Body water; Extracellular fluid; Gynaecological surgery; Lymphoedema; Postoperative complications 


\section{Abbreviations}

$\begin{array}{ll}\text { BCM } & \text { body cell mass } \\ \text { BIA } & \text { bioelectrical impedance analysis } \\ \text { BIS } & \text { bioelectrical impedance spectroscopy } \\ \text { C } & \text { capacitance } \\ \text { CLI } & \text { capillary leak index } \\ \text { ECV } & \text { extracellular fluid volume } \\ \text { FFM } & \text { fat free mass } \\ \text { FFMI } & \text { fat free mass index } \\ \text { FM } & \text { fat mass } \\ \text { FMI } & \text { fat mass index } \\ \text { HGS } & \text { hand grip strength } \\ \text { ICV } & \text { intracellular fluid volume } \\ \text { LLL } & \text { lower limb lymphoedema } \\ \text { LO } & \text { lymphoedema } \\ \text { MF-BIA } & \text { multifrequency BIA } \\ \text { NRI } & \text { nutrition risk index } \\ \text { NRS } & \text { nutrition risk score } \\ \text { NSCLC } & \text { non-small cell lung cancer } \\ \text { PEF } & \text { peak expiratory flow } \\ \text { PhA } & \text { phase angle } \\ \text { R } & \text { resistance } \\ \text { SCCHN } & \text { squamous cell carcinoma head/neck } \\ \text { SD } & \text { standard deviation } \\ \text { SF-BIA } & \text { single frequency BIA } \\ \text { SGA } & \text { subjective global assessment } \\ \text { SPhA } & \text { standardised phase angle } \\ \text { total body water } \\ \text { reactance } \\ \text { impedance }\end{array}$




\section{Introduction}

Postoperative recovery without complications and long-term adverse side effects is the preference of all patients and the health care providers. However, for many reasons this goal is not always achievable, but substantial measures should be taken to minimise the risks for peri- and postoperative complications and adverse side effects of the treatment. Although many risk factors for postoperative complications and long-term adverse side effects are known, there is still a need for simple methods that, perioperatively, can predict and thus make it possible to prevent or restrict the development of these unwanted qualities.

During the past two decades, bioelectrical impedance analysis (BIA) has become a useful tool in clinical research. As a non-invasive method, it provides an estimation of total body fluid volume (TBV) expressed as fat-free mass (FFM). Through its geometrically based algorithm, BIA gives information on extracellular fluid volume (ECV) and intracellular fluid volume (ICV).

Body composition and hydration status contain valuable information about the patients’ wellbeing as several medical conditions are accompanied by changes in TBV, body cell mass (BCM), fat mass (FM), FFM, ECV and ICV.

In this descriptive systematic review, we aimed to summarise the contemporary evidence of use of BIA in gynaecological surgical patients in studies published between 2008 and 2018. In particular, we highlighted the use of BIA for detection and prediction of lymphoedema and its use perioperatively for prediction of postoperative recovery. Where gynaecological studies have yet to be conducted in this field, we intended to give a theoretical reasoning regarding how the BIA method could be applicable in this patient category. 


\section{Material and Methods}

The PubMed, Scopus, Web of Science, MEDLINE, the Cochrane Library and Google Scholar databases were searched for articles published during the period January 2008 - April 2018. The reference lists in all identified relevant articles and reviews were searched for additional published studies concerning the topic of bioelectrical impedance.

Studies were included based on the following criteria: 1) studies with whole body bioelectrical impedance analysis, 2) an adult study population, 3) covering gynaecological patients and using the bioelectrical impedance method, 4) a gynaecological study population or a clinical setting that can be applied to the gynaecological patient.

The search terms used included: bioelectrical impedance analysis, bioelectrical analysis, BIA, BIS, BIVA, MF-BIA, phase angle, fluids, electrolytes, hydration, dehydration, overhydration, hypohydration, sodium, hypernatraemia, female body composition, extracellular volume, intracellular volume, ECV, ICV, intracellular fluid, extracellular fluid, perioperative patient, perioperative gynaecological patient, gynaecological cancer, gynaecological surgery, operative hysteroscopy, lymphoedema, lower limb lymphoedema, lymphatic overload, lower abdomen surgery, postoperative nausea and vomiting, postoperative recovery, oxidative stress. AND/OR was used between the different search terms.

Where no gynaecological studies were found, articles covering abdominal, urological or breast surgery/cancer were used instead and a theoretical reasoning was used to apply this to the gynaecological settings. Only papers published in English were included in the review. Articles covering case reports, paediatric study populations, or articles which did not declare which BIA tool or which frequencies were used in the bioelectrical impedance analysis, were excluded.

When no equation model was given to the impedance values, the manufacturer's own bioimpedance system was assumed to be used. These systems are named 'manufacture' throughout the paper.

\section{Bioelectrical impedance analysis (BIA)}


There are several types of BIA instruments available on the market. The different instruments used in this review (Table 1) are single frequency-BIA (SF-BIA) (1-6), multifrequency-BIA (MFBIA)(7-9), and bioelectrical impedance spectroscopy (BIS) (10-16).

The theoretical principle is the same for all BIA instruments. Electrodes are attached to the body in a standard tetrapolar arrangement following a standardised protocol, and a weak electrical current is passed through the human body. BIA divides the body into five cylinders; trunk, upper and lower extremities (Figure 1). Several parameters can be calculated. Impedance (Z) is the frequency-dependent opposition by the conductor (the human body), to the flow of the electric current (17). Geometrically $\mathrm{Z}$ is a vector composed of resistance (R) and reactance (Xc), both frequency-dependent parameters (18). $\mathrm{R}$ is the opposition to the flow of current when passing through the body and is inversely proportional to the amount of water. The assumption is that low frequencies cannot penetrate cell membranes and, thus, measure the ECV (15), while a high frequency current passes through both intracellular and cellular spaces allowing for quantification of TBV (19). Xc is the delay in conduction caused by cell membranes, tissue interfaces and nonionic substances and is related to the structure and function of cell membranes (20). Capacitance (C) is the function of the reactance that arises when cell membranes store a portion of the electrical current. This temporary storage creates the phase angle (PhA) (17). PhA represents the cellular integrity (Figure 1) and is the direct ratio between $\mathrm{Xc}$ and $\mathrm{R}(21)$. PhA is quantified geometrically as the angular transformation of the ratio of the arc tangent of reactance to resistance expressed in degrees (Fig $23 \mathrm{~A}$ and B) (3). PhA is calculated by; $P h A=(X c / R) x(180 \% \pi)(2,5,6)$. The standardised $\mathrm{PhA}(\mathrm{SPhA})$ is adjusted for sex and age, and is calculated by; $\operatorname{SPhA}=$ (observed $\mathrm{PhA}-$ mean $\mathrm{PhA}$ )/SD of the PhA. The mean of $\mathrm{PhA}$ is derived from the relationship between resistance and reactance. Negative values of the SPhA represent values below the reference mean (3).

The $\mathrm{PhA}$ is interpreted as a direct measure of cell stability and is an indicator of cell membrane integrity. A low $\mathrm{PhA}$ suggests cell death or decreased cell integrity, while a high $\mathrm{PhA}$ implies a large quantity of intact cells (2). Thus, PhA may be seen as a measure of tissue damage. 
The suggested reference values for PhA range from 4.8 to 8, depending on gender and age (22). $\mathrm{PhA}$ has been used as a predictor of skeletal muscle mass (23), as a prognostic factor in cancer patients (4) and as a predictor of postoperative complications (9).

\section{Lymphoedema}

Lymphoedema (LO) is the swelling that occurs when protein-rich lymph fluid accumulates in the interstitial space, resulting from damaged or blocked lymphatic vessels that inhibit the drainage of fluid from tissues (24). The subcutaneous accumulation of lymph fluid is the first sign of LO development and is characterised by an increase in the ECV (13). As LO progresses, the fluid increases in protein content with cellular infiltration, eventually developing tissue fibrosis and fat deposition in the skin and subcutaneous tissue. As a result, the overall limb volume may continue to increase, but the fluid content decreases proportionately (14).

LO is a chronic and progressive condition that may be physically and psychosocially disabling and can cause substantial impact on the quality of life. Ultimately, established LO may be a serious and lethal condition causing septic shock and tissue transformation to liposarcoma. Treatment of LO at an early stage is therefore important in order to prevent or reduce the severe long-term effects (25.)

Lower limb lymphoedema (LLL) is a common complication after gynaecological cancer surgery. The primary surgical treatment of early-stage gynaecological cancers very often includes an evaluation of the local and regional lymph nodes by means of a lymphadenectomy in order to detect metastases. In early-stage cancers, the spread of the cancer to the lymph nodes is a very strong negative prognostic factor for survival. Moreover, spread to the lymph nodes also indicates the need for adjuvant oncological therapy. Women with early-stage gynaecological cancer who do not have lymph node metastases generally have an excellent prognosis and become long-term survivors. It is important to find methods to predict the development of LLL since not all women with gynaecological cancers who have had surgery with lymphadenectomy develop LLL. 
Consequently, it may be possible to anticipate who needs prophylactic measures to prevent the progress of an established early LLL.

\section{Fluid measurement}

BIA is valuable in the clinical setting since it is able to assess TBV in subjects even without significant fluid or electrolyte abnormalities (17). Patients undergoing anaesthesia and surgery routinely receive various intravenous fluid infusions to achieve haemodynamic stability during surgery. The physiological stress response to surgery induces fluid retention, inflammation and catabolism (26). The perioperative fluid balance is an important factor affecting surgical outcomes and postoperative recovery. Changes affecting both ECV and ICV are visible already on postoperative day 1 (9), and even a moderate increase seems to increase the risk of postoperative complications (27). Protocols for enhanced recovery after surgery recommend salt and water restriction and near zero fluid balance to improve postoperative outcomes (28). However, it is unclear whether restrictive or zero fluid balance is applicable for all major abdominal surgeries (29). Hence, a reliable clinical detection method of perioperative hydration status may be valuable for improving postoperative recovery. 


\section{Results}

The selection of articles is summarised in the flow chart (Figure 3). Thirty-seven articles were evaluated. Studies on BIA within gynaecological surgical patients $(n=16)$ were divided into three categories: BIA and lower limb lymphoedema (n=7), BIA and perioperative hydration measuring ( $\mathrm{n}=3$ ), and $\mathrm{PhA}$ as a prognostic factor in cancer survival and as predictive for postoperative complications $(n=6)$.

The bioimpedance method and development of lymphoedema

Rather few studies have been published concerning BIA and LLL following gynaecological surgery. Table 2 summarises the studies, which used BIA to detect LLL, published between 2008 and 2018. Of the seven reviewed articles, four were from the same research group,(10-12,14) and only two concerned women after gynaecological surgery $(7,13)$. BIS was the most commonly used BIA method; only one study used MF-BIA (7). Previous studies performed on upper limb LO after breast cancer surgery and axillary node dissection have proposed BIS to be the preferred BIA method to detect LO (30) with a sensitivity of 73\% and a specificity of $84 \%$ (31).

Hayes et al. suggested that BIS was less capable of detecting LO in the genital area following vulvar/vaginal surgery (13). However, the equipment used in their study was not able to assess body fluids in the central compartment of the body (13), thus was not able to detect LO that develops in the pelvic area. In contrast, the MF-BIA has been suggested to detect LLL even before subjective symptoms appear (7).

There are several ways to interpret and estimate LLL by using the BIA parameters. The ECV/ICV ratio $(11,15)$ and ECV/TBV (7) ratio have been used to detect LLL. Another method has been to compare the ratio of the ECV in the respective lower extremities, $\mathrm{ECV}_{1} / \mathrm{ECV}_{2}(10)$. The ECV of the upper extremity can also serve as a reference value when investigating the lower limbs (12). An impedance ratio of ECV/ICV exceeding 1.136 has been suggested as reference value for the presence of LLLA (15). 
Five of the studies had a cross-sectional study design $(10-12,14,15)$. The two studies covering the development of LLL after gynaecological surgery had a prospective study design. The measurements were performed preoperatively and during postoperative day 7 (7) or at scheduled follow-ups up to 24 months (13). The patients who had undergone lymphadenectomy had an increased ECV/TBV in the lower limbs and trunk compared to patients without lymphadenectomy in the study that took the measurements on postoperative day 7 (7). Hayes et al. found that $37 \%$ of the women in their study at the 24-month follow-up had evidence of LLL as assessed by BIS. At the same time, the self-report of LLL was $45 \%$ (13). LLL was considered to be present when the BIS ratio of impedance at zero frequency of the arm/leg exceeded one standard deviation of the mean of normative ratios (13).

None of the studies have looked at the predictive value of BIA for prediction of LO/LLL.

\section{Fluid measurements and BIA}

The articles published on BIA covering perioperative fluid measurements are summarised in Table 3. Of the three examined articles, two used a gynaecological study population $(8,16)$ and one used a study population with hepato-pancreato-biliary disease (9). The latter study was included because of the similarity with ovarian cancer regarding the feature of occurrence of ascites. Ascites and fluid retention were the most common postoperative complications, and the finding of an increased ECV/TBV suggested a possible causality for the development of these complications (9).

In all studies, the extracellular fluid compartment increased postoperatively $(8,9,16)$. The TBV and the ECV were increased one month postoperatively after both benign and malign gynaecological surgery, although the increase was more pronounced after surgery involving lymphadenectomy (8). The perioperative fluid balance significantly correlated with changes in the ECV but not in the ICV (16). Interestingly, the capillary leak index (CLI) (the C-reactive protein over albumin concentration multiplied by one hundred) was also found to be a significant predictor of changes in the ECV (16). The rise in the CLI has been shown to be a predictor of poor prognosis 
in ovarian cancer patients. This may be due to the association between the CLI and inflammation (32).

The phase angle as a prognostic tool in the clinical setting

Another clinically useful application of the BIA method seemed to be the mathematically derived $\mathrm{PhA}$. It is calculated from $\mathrm{R}$ and $\mathrm{Xc}$ at $50 \mathrm{kHz}$, and therefore the SF-BIA was the only tool used in the examined articles (Table 4).

In the reviewed articles, the PhA was investigated as a prognostic factor for mortality in patients with breast cancer (2), advanced cancer $(1,5)$, gastrointestinal cancer $(4)$, or as a predictor for postoperative complications after elective gastrointestinal surgery(6) and after elective cancer surgery (3). None of these studies was carried out with solely gynaecological study populations. Norman et al. had gynaecological patients (20/399) in their population but the results were not stratified into tumour groups (4).

All the articles dealing with cancer concluded that a lower $\mathrm{PhA}$ at baseline indicated poorer prognosis. However, one study found that an increase in PhA during fluid therapy also predicted shorter survival (1). None of the studies presented a consensus of the cut-off value of PhA. The values suggested ranged between; $\mathrm{PhA}>6^{\circ}$ (6), $\mathrm{PhA}>5.6^{\circ}(2), 4^{\circ}-12^{\circ}(5)$, and for $\mathrm{SPhA} ;>0^{\circ}(3,4)$. Two studies used SPhA $(3,4)$, of which one study investigated the association between SPhA and survival. The study by Norman et al. found the SPhA ranged from $-5.52^{\circ}$ to $3.09^{\circ}$, where higher values reflected better six-month prognosis for mortality in cancer patients (4). Härter et al. looked at oncological surgical patients and the occurrence of severe postoperative complications (3). Patients in their study who experienced postoperative complications had a significantly lower SPhA $\left(-0.71^{\circ}\right)$ than patients without postoperative complications $\left(0.41^{\circ}\right)$.

Two studies investigated PhA as a predictor for the development of postoperative complications. The PhA correlated significantly with the occurrence of postoperative complications in the univariate analysis $(3,6)$ but the significance disappeared in the multivariate analysis $(6)$. 


\section{Comments}

BIA seemed to offer a simple non-invasive way of evaluating the occurrence of LLL, measuring fluid status on a daily basis in the perioperative patient, being predictive for complications postoperatively, and a prognostic factor following cancer treatment in the gynaecological patient.

The clinical diagnosis of lymphoedema requires physical symptoms that are clinically detectable and usually incorporates the identification of the symptomatic characteristics of LO in stage 2 with a firm non-pitting oedema. LO following surgery is preferably treated with physiotherapy; a regimen of exercises, compression bandaging and massage (33). The BIA had high reliability for detecting lymphoedema in the lower extremities (34). As LLL has a better treatment prognosis the earlier it is detected, the BIA may be a useful tool for detecting lymphoedema early in the course. One month after gynaecological surgery with lymphadenectomy the ECV was shown to be significantly increased compared to benign surgery without lymphadenectomy.(8)

There are several ways to interpret the BIA data as an expression of LLL. When LLL is presented unilaterally, the ECV from the lower limb can be compared with that of the contralateral limb. However, LLL due to gynaecological surgery is often presented bilaterally when lymphadenectomy has been conducted on both pelvic sidewalls or in the groins. To address this issue of LLL, baseline bioelectrical impedance values should therefore always be measured preoperatively. In the absence of baseline measurements, the impedance values from the upper limb have been suggested to serve as reference values.

The BIA devices do not seem to be interchangeable and significant differences have been found between instruments in both measurements of absolute impedance and limb impedance ratios (30). Advanced lymphoedema might be falsely measured by BIA. A persistent accumulation of extracellular lymphatic fluid promotes the proliferation of adipocytes and the deposition of collagen fibres which causes fibrosis. These tissues are non-conductive and can thus interfere with the measurement of impedance (14). 
Fluid therapy is guided in the clinical practice by parameters such as blood pressure, heart rate, and diuresis. These parameters are also affected by variables not related to the circulatory status, including pain, body temperature, physiological and psychological stress, as well as use of anaesthetic and analgesic drugs. Fluid retention is common during the postoperative course, and can occur despite a negative intraoperative fluid balance, a strict perioperative fluid restriction, an early mobilisation and an encouraged shift from intra venous to oral fluids (35). The BIA has been shown to be an early informative and sensitive marker for perioperative fluid balance with significant correlations with changes in the ECV but not in the ICV (16). It has been suggested to be more accurate than the serum NT-pro-BNP for detecting peripheral oedema (36) and useful in the estimation of body fluids in connection with hyponatraemia (37).

The phase angle represents the integrity of cellular membranes (21) and has been used as a marker for clinical prognosis in cancer patients and for postoperative complications. Reference values of $\mathrm{PhA}$ have been estimated to range between $4-12^{\circ}$. However, a standardised reference value is yet to be presented. A PhA lower than $6^{\circ}$ indicated worse prognosis (6) and, generally, the $\mathrm{PhA}$ was slightly lower in women than in men due to women's lower muscle mass, and $\mathrm{PhA}$ increased with obesity due to the increased number of adipocytes (4).

The strength of this review is the focus on the application of BIA for the gynaecological surgical patient. The most used BIA method was the SF-BIA used in 16 of the studies. None of the BIA techniques seemed to be superior to another in terms of body fluid estimations, patient safety or ease of use. However, each of the different BIA methods has its own advantages and disadvantages.

The review also showed some weaknesses. We could not strictly include studies with a gynaecological study population. Only three of the included studies had entirely gynaecological surgical populations. Instead, we chose studies that evaluated mortality in cancer patients, investigating postoperative complications in surgical cancer patients and in surgical gastrointestinal patients, because the general clinical outline and pathologies were assumed to be similar to general 
surgical gynaecological patients. The bioelectrical impedance measurements have been suggested to depend on age, gender and body mass characteristics, and thus, different study populations and mixed gender probably limit the extrapolation of the results. BIA is still a rather new and relatively unexplored method and there is as yet no agreement on standardisation of the method or references with limits for deviant values. Moreover, the method requires reference values from a healthy population to be established. To date, BIA has been explored in several medical conditions but the interpretation of the BIA parameters in daily clinical practice is still uncertain. It seems that different types of apparatus cannot be interchanged with each other as the setting and mathematical formulas programmed vary, to give parameters such as impedance, reactance and resistance. This has an impact on the absolute values, as the reference values are different in all the studies investigated in this review. In this review, the apparatuses had been sorted according to BIA frequencies (SF-BIA, MF-BIA, BIS) but not according to brands. This may be seen as a limitation since different brands may vary in quality.

Two of the examined articles were retrospective (2,9), five had a cross-sectional (10$12,14,15)$, and nine were prospective $(1,3-8,13,16)$. The articles were chosen from a time period of 10 years because the techniques of BIA prior to 2008 are arguably not comparable with those used today.

\section{Conclusion}

There seems to be a wide range of promising applications for the BIA for predicting and eventually preventing clinical complications in the gynaecological surgical patient as listed below:

- $\quad$ BIA can detect lymphoedema at a subclinical level and may therefore be an important tool for diagnosing lymphoedema at an early stage. Early detection provides the opportunity to prevent, treat or reduce the progress of LO. However, in order to detect early development of LLL after gynaecological cancer surgery with lymphadenectomy the predictive value of consecutive measurements of BIA in the perioperative course remains to be investigated. 
- $\quad$ BIA studies have shown that the ECV increased more than the ICV postoperatively. The clinical impact of this merits further investigation concerning the possible association with the development of postoperative complications and long-term adverse side effects.

- $\quad$ The PhA can be used as an estimate of intracellular health and cell membrane integrity. This appears promising for measuring post-surgery inflammation and the occurrence of postoperative complications. 


\section{Acknowledgements}

The study was financially supported by Linköping University and Region Östergötland. 


\section{References}

1. Davis MP, Yavuzsen T, Khoshknabi D, Karafa MT. Bioelectrical impedance phase angle changes during hydration and prognosis in advanced cancer. Am J Hosp Palliat Care. 2009;26:180-7.

2. Gupta D, Lammersfeld C a, Vashi PG et al. Bioelectrical impedance phase angle as a prognostic indicator in breast cancer. BMC Cancer. 2008;8:249.

3. Härter J, Orlandi SP, Gonzalez MC. Nutritional and functional factors as prognostic of surgical cancer patients. Support Care Cancer. 2017;25(8):2525-30.

4. Norman K, Stoba N, Zocher D et al. Cutoff percentiles of bioelectrical phase angle predict functionality, quality of life, and mortality in patients with cancer. Am J Clin Nutr. 2010;92(3):612-9.

5. Navigante A, Morgado PC, Casbarien O. Relationship between weakness and phase angle in advanced cancer patients with fatigue. Support Care Cancer. 2013;21(6):1685-90.

6. Schiesser M, Kirchhoff P, Müller MK, Schäfer M, Clavien PA. The correlation of nutrition risk index, nutrition risk score, and bioimpedance analysis with postoperative complications in patients undergoing gastrointestinal surgery. Surgery. 2009;145(5):519-26.

7. Takeuchi M, Yoshizawa T, Kusaka Y, Furusawa Y, Nakamura Y. Detecting subclinical secondary lymphoedema using bioimpedance : A preliminary study. J Lymphoedema. 2013;8(2):16-20.

8. Ilhan TT, Uçar MG, Pekin AT et al. Does lymphadenectomy have influence on postoperative body fluid distribution? Eur J Obstet Gynecol Reprod Biol. 2016;212:182-5.

9. Chong JU, Nam S, Kim HJ, Lee R et al. Exploration of fluid dynamics in perioperative patients using bioimpedance analysis. J Gastrointest Surg. 2016;20(5):1020-7.

10. Suehiro K, Morikage N, Ueda K et al. Local echo-free space in a limb with lymphedema represents extracellular fluid in the entire limb. Lymphat Res Biol. 2017;16(2):187-192.

11. Suehiro K, Morikage N, Yamashita $\mathrm{O}$ et al. Echo-free space and the amount of extracellular fluid determined by bioelectrical impedance analysis of leg edema. Lymphat Res Biol. 2017;15(2):172-6.

12. Suehiro K, Morikage N, Harada T et al. Application of the L-dex score for the assessment of bilateral leg edema. Lymphat Res Biol. 2017;16(1):65-8.

13. Hayes SC, Janda M, Ward LC et al. Lymphedema following gynecological cancer: Results from a prospective, longitudinal cohort study on prevalence, incidence and risk factors. Gynecol Oncol. 2017;146(3):623-9.

14. Suehiro K, Morikage N, Yamashita O et al. Distribution of extracellular fluid in legs with venous edema and lymphedema. Lymphat Res Biol. 2016;14(3):156-61. 
15. Ward L, Winall A, Isenring E, Hills A et al. Assessment of bilateral limb lymphedema by bioelectrical impedance spectroscopy. Int J Gynecol Cancer. 2011;21(2):409-18.

16. Ernstbrunner M, Kostner L, Kimberger $\mathrm{O}$ et al. Bioimpedance spectroscopy for assessment of volume status in patients before and after general anaesthesia. PLoS One. 2014;9(10): e111139.

17. Kyle UG, Bosaeus I, Lorenzo AD De, M et al. Bioelectrical impedance analysis part I : Review of principles and methods. Clin Nutr. 2004;5(23):1226-43.

18. Mulasi U, Kuchnia AJ, Cole AJ el al. Bioimpedance at the bedside: Current applications, limitations, and opportunities. Nutr Clin Pract. 2015;30(2):180-93.

19. Buchholz AC, Bartok C, Schoeller DA. The validity of bioelectrical impedance models in clinical populations. Nutr Clin Pract. 2016;19(5):433-46.

20. Barbosa-Silva MCG, Barros AJD. Bioelectric impedance and individual characteristics as prognostic factors for post-operative complications. Clin Nutr. 2005;24(5):830-8.

21. Selberg O, Selberg ÆD. Norms and correlates of bioimpedance phase angle in healthy human subjects, hospitalized patients, and patients with liver cirrhosis. Eur J Appl Physiol. 2002;86(6):509-16.

22. Kyle UG, Genton L, Pichard C. Low phase angle determined by bioelectrical impedance analysis is associated with malnutrition and nutritional risk at hospital admission. Clin Nutr. 2013;32(2):294-9.

23. Kim JH, Choi SH, Lim S et al. Assessment of appendicular skeletal muscle mass by bioimpedance in older community-dwelling Korean adults. Arch Gerontol Geriatr. 2014;58(3):303-7.

24. Grada AA, Phillips TJ. Lymphedema. J Am Acad Dermatol. 2017;77(6):1009-20.

25. Beesley VL, Rowlands IJ, Hayes SC,et al. Incidence, risk factors and estimates of a woman's risk of developing secondary lower limb lymphedema and lymphedema-specific supportive care needs in women treated for endometrial cancer. Gynecol Oncol. 2015;136(1):87-93.

26. Scott MJ, Baldini G, Fearon KCH,et al. Enhanced Recovery after Surgery (ERAS) for gastrointestinal surgery, part 1: Pathophysiological considerations. Acta Anaesthesiol Scand. 2015;59(10):1212-31.

27. Nilsson L, Wodlin NB, Kjølhede P. Risk factors for postoperative complications after fasttrack abdominal hysterectomy. Aust N Z J Obstet Gynaecol. 2012 ;52(2):113-20.

28. Lassen K, Coolsen MME, Slim K et al. Guidelines for perioperative care for pancreaticoduodenectomy: Enhanced recovery after surgery (ERAS) society recommendations. World J Surg. 2013;37(2):240-58.

29. Eng OS, Melstrom LG, Carpizo DR. The relationship of perioperative fluid administration to 
outcomes in colorectal and pancreatic surgery: A review of the literature. J Surg Oncol. 2015;111(4):472-7.

30. Zanten M Van, Piller N, Ward LC. Inter-changeability of impedance devices for lymphedema assessment. Lymphat Res Biol. 2016;14(2):88-94.

31. Bundred NJ, Stockton C, Keeley V et al. Comparison of multi-frequency bioimpedance with perometry for the early detection and intervention of lymphoedema after axillary node clearance for breast cancer. Breast Cancer Res Treat. 2015;151(1):121-9.

32. Liu Y, Chen S, Zheng $\mathrm{C}$ et al. The prognostic value of the preoperative c-reactive protein / albumin ratio in ovarian cancer. BMC Cancer. 2017;285(17):4-11.

33. Biglia N, Librino A, Ottino MC at al. Lower limb lymphedema and neurological complications after lymphadenectomy for gynecological cancer. Int J Gynecol Cancer. 2015;25(3):521-5.

34. Hidding JT, Viehoff PB, Beurskens CHG et al. Instruments for measuring of lymphedema: Systematic review. Phys Ther. 2016;96(12):1965-81.

35. Cagini L, Capozzi R, Tassi V et al. Fluid and electrolyte balance after major thoracic surgery by bioimpedance and endocrine evaluation. Eur J Cardio-thoracic Surg. 2011;40(2).

36. Massari F, Iacoviello M, Scicchitano P et al. Accuracy of bioimpedance vector analysis and brain natriuretic peptide in detection of peripheral edema in acute and chronic heart failure. Hear Lung J Acute Crit Care. 2016;45(4):319-26.

37. Kim JS, Lee JY, Park H, Han BG, Choi SO, Yang JW. Estimation of body fluid volume by bioimpedance spectroscopy in patients with hyponatremia. Yonsei Med J. 2014;55(2):482-6. 
Table 1. Different bioelectrical impedance techniques used in studies between 2008 - 2018. The common theory for all methods described in the table: An alternating current is applied, typically at the wrist and the ankle of the patient, and the response is measured as resistance at reactance. At low frequencies $<50 \mathrm{kHz}$ the electrical current cannot penetrate cell membranes and therefore predict ECV.

Bioelectrical impedance measurements Concept

Reference

SF-BIA: single frequency BIA

Typically use of $50 \mathrm{kHz}$. Where articles did not specify if they used single- or multifrequency, methods using frequency at only $50 \mathrm{kHz}$ were categorized

SF-BIA.

MF-BIA: multifrequency BIA

Typically use of 5,50 and $100 \mathrm{kHz}$

Higher frequency $>50 \mathrm{kHz}$ can penetrate cell membranes and be used to

estimate ICV

ECV and ICV are calculated using the Hanai and Cole model rather than

BIS: Bioelectrical impedance spectroscopy

regression equations to predict body composition. These models allows separation of fluid overload from the muscle mass. The term spectroscopy is used because BIS utilise a spectra of frequencies.

ECV: extra cellular volume; ICV: intra cellular volume 
Table 2. Lower limb lymphoedema in the clinical setting measured by bioelectrical impedance analysis.

Author

\begin{tabular}{|c|c|c|c|c|}
\hline $\begin{array}{c}\text { (Reference nr.) } \\
\text { Year }\end{array}$ & $\begin{array}{c}\text { BIA } \\
\text { method }\end{array}$ & BIA parameters and equation & Study method and objects & Main findings and Comments \\
\hline $\begin{array}{l}\text { Ward et al. } \\
(15) \\
2011\end{array}$ & BIS & $\begin{array}{l}E C V, I C V, R_{i}, R_{\infty} \\
R_{i}=\left(R_{0} \times R \infty\right) /\left(R_{0}-R \infty\right) \\
E C V / I C V=R_{i} / R_{0} \\
\text { ECV/ICV ratio calculated for each }\end{array}$ & $\begin{array}{l}\text { A cross-sectional study with patients clinically } \\
\text { diagnosed with bilateral LLL (women } n=37 \text {, } \\
\text { males } n=5 \text { ). } \\
\text { Healthy controls } \\
\text { (males } n=224 \text {; women } n=277 \text { ) }\end{array}$ & $\begin{array}{l}\text { ECV/ICV varied with age, sex, and limb dominance } \\
(p<0.001) \text {. No significant interaction between age } \\
\text { and limb dominance. ECV/ICV higher in both upper } \\
\text { and lower extremities of men compared to women ( } p \\
<0.001) \text {. }\end{array}$ \\
\hline
\end{tabular}

Takeuchi et al. MF-BIA ECV, ICV, TBV

(7)

2013

Suehiro et al

2016
BIS

\section{$\begin{aligned} \text { MF-BIA } & \text { ECV, ICV } \\ & \text { ECV/TBV }\end{aligned}$}

For healthy individuals a ratio of

$0.36-0.40$ was considered normal. Higher values indicated increased

TBV.

ICV, ECV

ICVIECV ratio
A prospective single-centre observational study of two groups of patients who underwent gynaecological surgery with $(n=$

12) and without lymphadenectomy ( $n=6)$. BIA measurements preoperatively and on postoperative day 7 .

A cross-sectional study of patients with $\mathrm{LO}$ ( $\mathrm{n}$ $=47)$ and patients with LVO $(n=33)$.

Duplex venous ultrasound

Subcutaneous tissue ultrasonography. Three protocols; protocol 2 and 3 on all patients (2) impedance in each leg normalized to the arm (3) impedance in the thigh and calf without normalization. Protocol 1 only performed in patients with unilateral leg oedema - impedance in the affected leg normalized to the contralateral leg.

BIA = bioelectrical impedance analysis; BIS = bioelectrical impedance spectroscopy; ECV = extra cellular volume; ICV = intra cellular volume; $\mathrm{LLL}=$ lower limb lymphoedema; $\mathrm{LO}=$ lymphoedema; $\mathrm{LVO}=$ leg with venous oedema; MF-BIA = multifrequency-BIA; $\mathrm{R}_{\mathrm{x}}=$ resistance; $\mathrm{TBV}=$ total body fluid volume. 
Table 2 continued. Lower limb lymphoedema in the clinical setting measured by bioelectrical impedance analysis.

Author

\section{(Reference nr.). BIA} Year method BIA parameters and equation Study method and objects Main findings and Comments

Hayes et al. BIS

(13) 2017 ECV $L O=$ the ratio of $Z$ at 0 frequencies of arm/legs exceeded one SD of the mean normative ratios.

\section{Suehiro et al.}

(12)

2017

\section{BIS}

ECV, ICV

ICV/ECV. Impedance in the oedematous leg normalized to the contralateral leg.

\section{Suehiro et al.}

BIS

(11)

Suehiro et al. BIS

(10) 2017

ECV of the affected limb compared

\section{ECV/ICV ratio.}

Resistance of ICV and resistance of $\mathrm{ECV}(\mathrm{Ri} / \mathrm{Re})$ ratio.

L-DEX = comparing ECV of affected leg to the unaffected when lymphoedema presented unilateral.

\section{ECV} with the contralateral normal limb. The ratio then compared with a normal population where LO defined as $>3$ SD greater than the mean.
A prospective study of women newly diagnosed with gynaecological cancer $(n=408)$.

Self-reporting measures

Objective measurement

Preoperative baseline measurements, follow up at six weeks, three months, 6, 12 and 1524 months.

A cross-sectional study of patients with unilateral leg oedema ( 3 males, 30 women) compared to a healthy population (13 males, 29 women). Lymphoedema index (L-DEX score)

A cross-sectional study of patients with LVO (9 males and 28 females) and with LLL (9 males and 41 females).

Subcutaneous tissue ultrasonography.

A cross-sectional study of patients with LLL (7 males, 38 women) and patients with LO (2 males, 38 women) in the arms. Immediately after the BIA measurements were taken the limbs were scanned with B-mode scan of the subcutaneous tissue with ultrasonography. Legs were scanned at eight points. SEFS was graded from 0 to 2 , where grade $0=$ no echo-

BIA = bioelectrical impedance analysis; BIS = bioelectrical impedance spectroscopy; ECV = extra cellular volume; ICV = intra cellular volume; $\mathrm{LLL}$ = lower limb lymphoedema; $\mathrm{LO}=$ lymphoedema; $\mathrm{LVO}=$ leg with venous oedema; $\mathrm{R}_{\mathrm{x}}=$ resistance; $\mathrm{SD}=$ standard deviation; $\mathrm{SEFS}=$ subcutaneous echo-free space; $\mathrm{VO}=$ venous oedema, 
Table 3: Bioelectrical impedance articles covering perioperative hydration measurements. Studies published between 2008 - 2018.

Author

(Reference nr) BIA

Year method

Ernstbru BIS

BIA parameters and equation ICV, ECV, TBV.

et al.

Manufacturers Cole model.

(16)

2014

\section{Chong et al. MF-BIA TBV, ECV, ICV, ECV/TBV}

(9)

2016

Ilhan et al. MF-BIA ECV, ICV, TBV.

(8)

2017
Study method and objects

A prospecptive study of BIA measurements

directly before and after standardized general

anesthesia in women ( $n=71)$ undergoing

gynaecological surgery (laparotomy,

laparoscopic, vaginal).

Preoperative measurement

Biochemistry

Body mass index

Capillary leak index defined as the ratio $\mathrm{C}$ reactive protein over serum albumin.

A retrospective study of perioperative fluid dynamics. Patients undergoing surgery for heapto-pancreato-biliary disease $(n=36)$.

Fluid input, urine output, skin turgor.

BIA measurments 1 day preoperatively, immediatley after surgery. Patients stratified as balanced $(\leq 500 \mathrm{~mL})$ or imbalanced $(>500 \mathrm{~mL})$ calculated net fluid status.

A prospective study of on fluid distribution after lymph node dissection (malignant $(n=92))$ or benign ( $n=89)$ gynaecological conditions). Measurements were performed on the date of hospitalization, at $24 \mathrm{hrs}, 1$ month post-surgical intervention
Main findings and comments

Routine intraoperative fluid administration resulted in a significant and clinically meaningful increase in the extracellular compartment. There was a significant positive correlation between net perioperative fluid balance and changes in pre- to postoperative ECV, $\mathrm{r}^{2}$ $=0.65, p<0.001$

Fluid imbalanced group showed postoperative increases of ECV $(p=0.001)$, ICV $(p=0.012)$, ECV/TBV $(p=0.019)$ compared to baseline. More postoperative complications were found in the imbalanced group. Ascites and fluid collections were the most common postoperative complications.

TBV was significantly increased 1 month after surgery in both malign and benign groups. ECV was significantly higher and ICV significantly lower in the malign group than in the benign group. No correlation to number of lymph nodes removed. Radical malign gynaecological surgery including lymph node dissection had a greater effect on TBV than surgery performed for benign conditions.

BIA = bioelectrical impedance analysis; BIS = bioelectrical impedance spectroscopy; ECV= extra cellular volume; ICV= intra cellular volume;

MF-BIA = multifrequency-BIA; TBV = total body fluid volume. 
Table 4: Bioelectrical impedance as a prognostic factor. In articles published between 2008 - 2018.

Author

(Reference $\mathrm{nr}$ )

BIA

\begin{tabular}{ccc}
$\begin{array}{c}\text { (Reference } \mathrm{nr}) \\
\text { Year }\end{array}$ & $\begin{array}{c}\text { BIA } \\
\text { method }\end{array}$ & $\begin{array}{c}\text { parameters } \\
\text { and equation }\end{array}$ \\
\hline Gupta et al. & SF-BIA & R, Xc, PhA
\end{tabular}

(2)

2008 SF-BIA

A

Study method and objects breast cancer patients $(n=259)$.

BIA measurement

Nutritional assessment

Survival defined from the first visit to the

hospital and the date of death, Kaplan-

Meier.

Schiesser et al. SF-BIA R, Xc, FFM, A prospective study of the occurrence of

(6)

ECV, PhA

2009

Davis et al.

SF-BIA

(1)

2009

PhA, R, Xc,

TBV, ECV,

ICV.

postoperative complications. Patients admitted for elective gastro-intestinal surgery ( $n=102$ men, $n=98$ women) age 18 to 85 years.

Preoperative screening and 5-months follow-up.

NRS and NRI

\section{A prospective observational study of}

continuous hydration as treatment. Patients $(\mathrm{n}=20$ women, $\mathrm{n}=30$ men) with advanced cancer (pancreatic, lung, breast, renal, colon and gastric cancer)

BIA daily on three consecutive days during ongoing hydration.

Patient-reported weight loss

Vital signs (body temperature, pulse \&

respiratory rate, blood pressure).

Physical examination (skin turgor, mucus membranes, peripheral oedema).
Presented cutoff values PhA The median PhA was 5.6. Those with a PhA $<5.6$ had a median survival of 23.1 months, while those with PhA $>5.6$ had a median survival of 49.9 months $(p=0.031)$.

$\operatorname{PhA}>5.6^{\circ}$

$25 \%$ post-operative complications.

$28,5 \%$ with $\mathrm{PhA}<6^{\circ}$.

Only NRS and malignancy were prognostic factors for the development of complications, odds ratios of 4.2, (1.2 $14.8,95 \% \mathrm{Cl})$ and $5.6(2.2-14.395 \% \mathrm{Cl})$.

$\mathrm{NRI}$, based on s-albumin concentration and weight loss, identified patients at risk for postoperative complications.

A higher PhA on day 1 predicted longer survival. An increase in PhA during hydration predicted shorter survival. PhA did not correlate with vital signs, the presence or absence of oedema, or day 1 potassium, sodium, chloride, creatinine or haemoglobin (Spearman correlation

coefficient $95 \% \mathrm{Cl}$ ).

A positive correlation was found between ECV/ICV and salbumin on day 1 .

PhA was inversely correlated with ECV/ICV each day $(p<$ $0.001)$ and inversely correlated with R on each day $(p<$

$0.05)$ except day $3(p=0.76)$.
$\mathrm{PhA}>6^{\circ}$

\footnotetext{
Blood chemistry.

BIA = bioelectrical impedance analysis; ECV = extra cellular volume; FFM = fat free mass; HGS = hand grip strength; ICV = intra cellular

volume; NRI = nutrition risk index; NRS = nutrition risk score; $\mathrm{PEF}=$ peak expiratory flow; $\mathrm{PhA}=$ phase angle; $\mathrm{R}=$ resistance; $\mathrm{SF}-\mathrm{BIA}=$ single frequency BIA; SMM = skeletal muscle mass; SGA = subjective global assessment; TBV = total body fluid volume; Xc = reactance.
} 
Table 4 continued: Bioelectrical impedance as a prognostic factor in articles published between 2008 - 2018 .

Author BIA

\begin{tabular}{ccc}
$\begin{array}{c}\text { (Reference } \mathrm{nr}) \\
\text { Year }\end{array}$ & $\begin{array}{c}\text { BIA } \\
\text { method }\end{array}$ & $\begin{array}{c}\text { parameters } \\
\text { and equation }\end{array}$ \\
\hline Norman et al. & SF-BIA & SPhA =
\end{tabular}

Norman et al. $\quad$ SF-BIA $\quad$ SPhA $=\quad$ A prospective study of cancer patients

(observed

PhA - mean

$\mathrm{PhA}) / \mathrm{SD}$ of

the PhA

women and $n=208$ men) $>60$ years of age.

Presented cut-

Tumour types gastrointestinal, head and neck

lung, urogenital, gynaecological,

neuroendocrine, others. Measurements

performed 48 hrs of hospital admission.

HGS, PEF, SPhA with a Z-score to determine

the individual deviations of the population

Navigante et al. SF-BIA

$\mathrm{R}, \mathrm{Xc}, \mathrm{PhA}$

average

(5)

A prospective study of the relationship between

cancer-related fatigue and PhA. Patients $(n=31$

men, $\mathrm{n}=10$ women) with locally advanced or

metastatic cancer (SCCHN, NSCLC)

Healthy control $(n=20)$

HGS. The grip work calculated as: (maximal

strength $\times 0.75) \times$ fatigue resistance.

Self-reporting fatigue scale.

Härter et al. SF-BIA PhA, SPhA $=$ A prospective study of surgical complications

(3)

(PhA - mean classified according to Clavien-Dindo. Patients

2017

$\mathrm{PhA}) / \mathrm{SD}$ of admitted for elective oncologic surgery $(n=34$

PhA.

males, $n=26$ women), head/neck ( $n=17)$,

unknown $(n=1)$, breast/gynaecology $(n=6)$, skin

$(n=5)$, gastrointestinal tract $(n=20)$ and

genitourinary $(n=11)$.

Negative SPhA values represent measures

below the reference mean.

HGS

One of the exclusion criteria: oedema in the lower limbs.

BIA = bioelectrical impedance analysis; PhA = phase angle; HGS = hand grip strength; NRS = nutrition risk score; NRI = nutrition risk index; NSCLC = non-small cell lung cancer; PEF = peak expiratory flow; $\mathrm{R}=$ resistance; SCCHN = squamous cell carcinoma head and neck; $\mathrm{SD}=$ standard deviation; SF-BIA = single frequency BIA; SPhA = standardised $\mathrm{PhA} ; \mathrm{Xc}=$ reactance. 


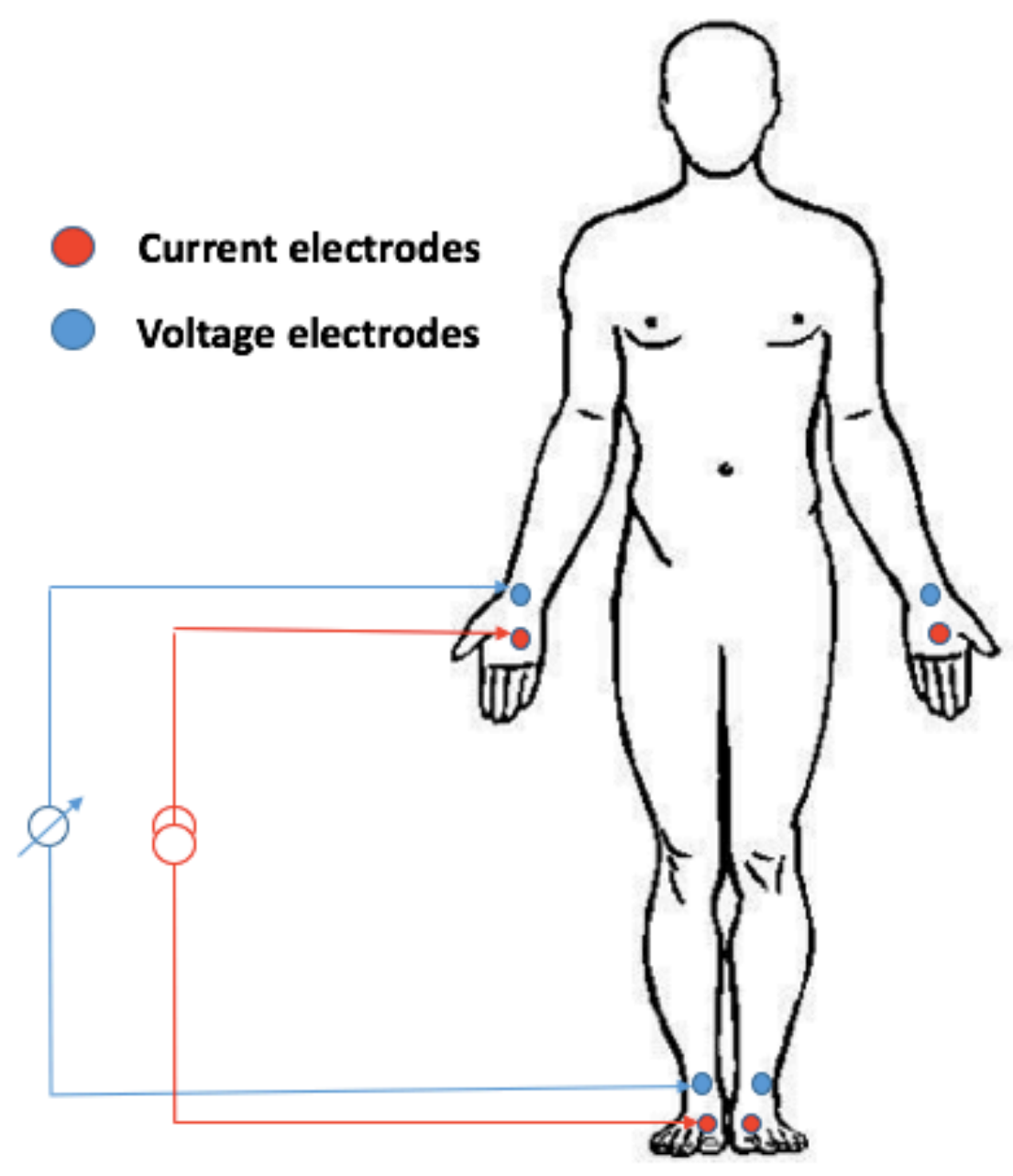

Figure 1. Standard palcement of electrodes for single- and multifrequency bioimpedance analysis. MF-BIA gives the impedance determinations at six different frequencies (1, 5, 50, 250, 500 and $1000 \mathrm{kHz}$ ) obtained on five body segments (both upper and lower extremities and the trunk). This gives the following volume measure: total body fluid volumer (TBV), extracellular volume (ECV), intracellular volume (ICV), and the phase angle (PhA). 
A

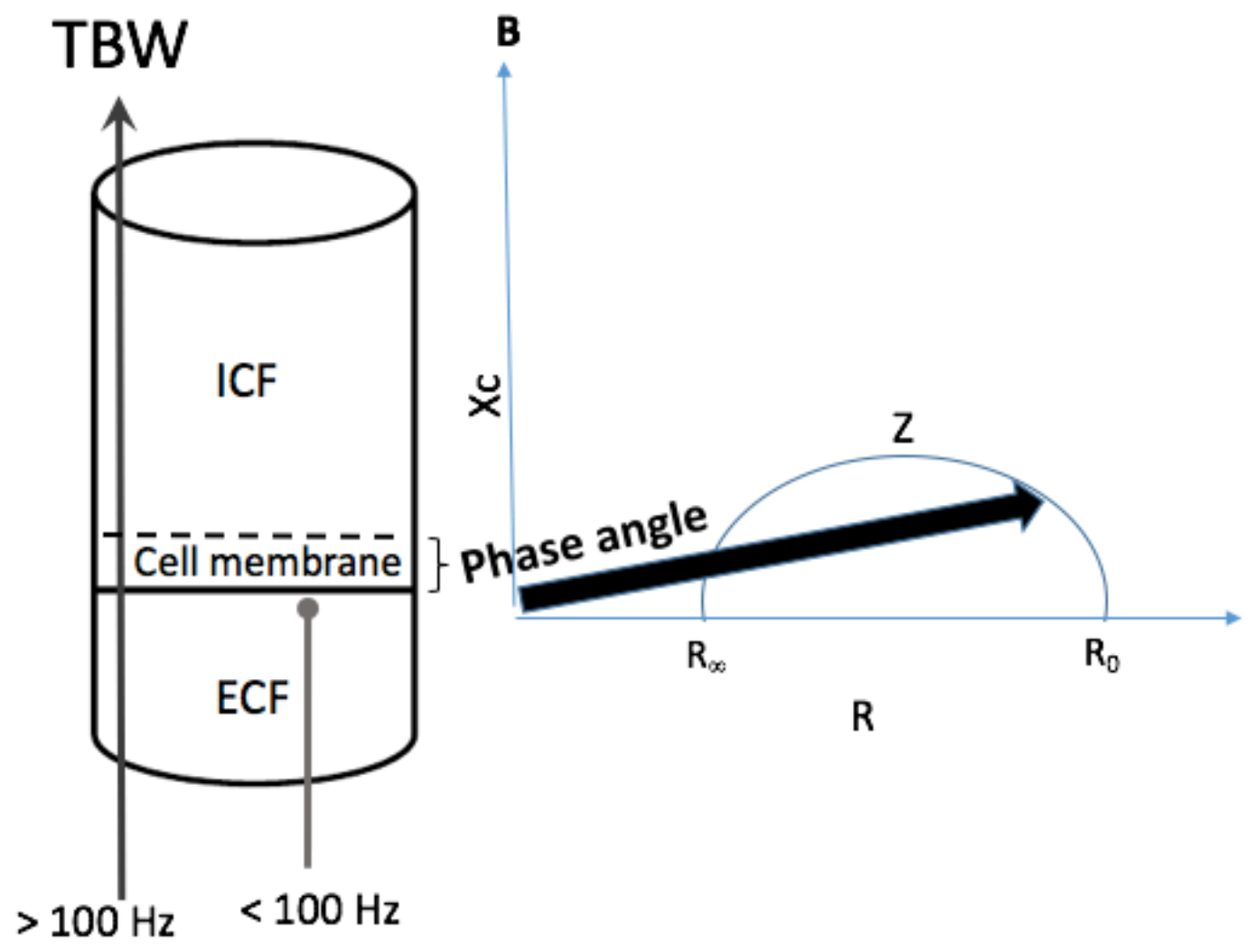

Figure 2. A) An electric current less than $100 \mathrm{kHz}$ is not able to pass through the cellular membrane and thus measures the extra cellular volume. Above $100 \mathrm{kHz}$ the electrical current can pass through the cellular membrane and thus gives the value of the total body fluid volume (TBV). When the electrical current passes through the cellular membrane there is a delay, which is the phase angle. The phase angle is calculated with the inverse trigonometric function. B) Diagram of the graphical presentation of $\mathrm{PhA}$, and the relationship with resistance $(\mathrm{R})$, reactance $(\mathrm{Xc})$, impedance $(\mathrm{Z})$ and the frequency applied. 


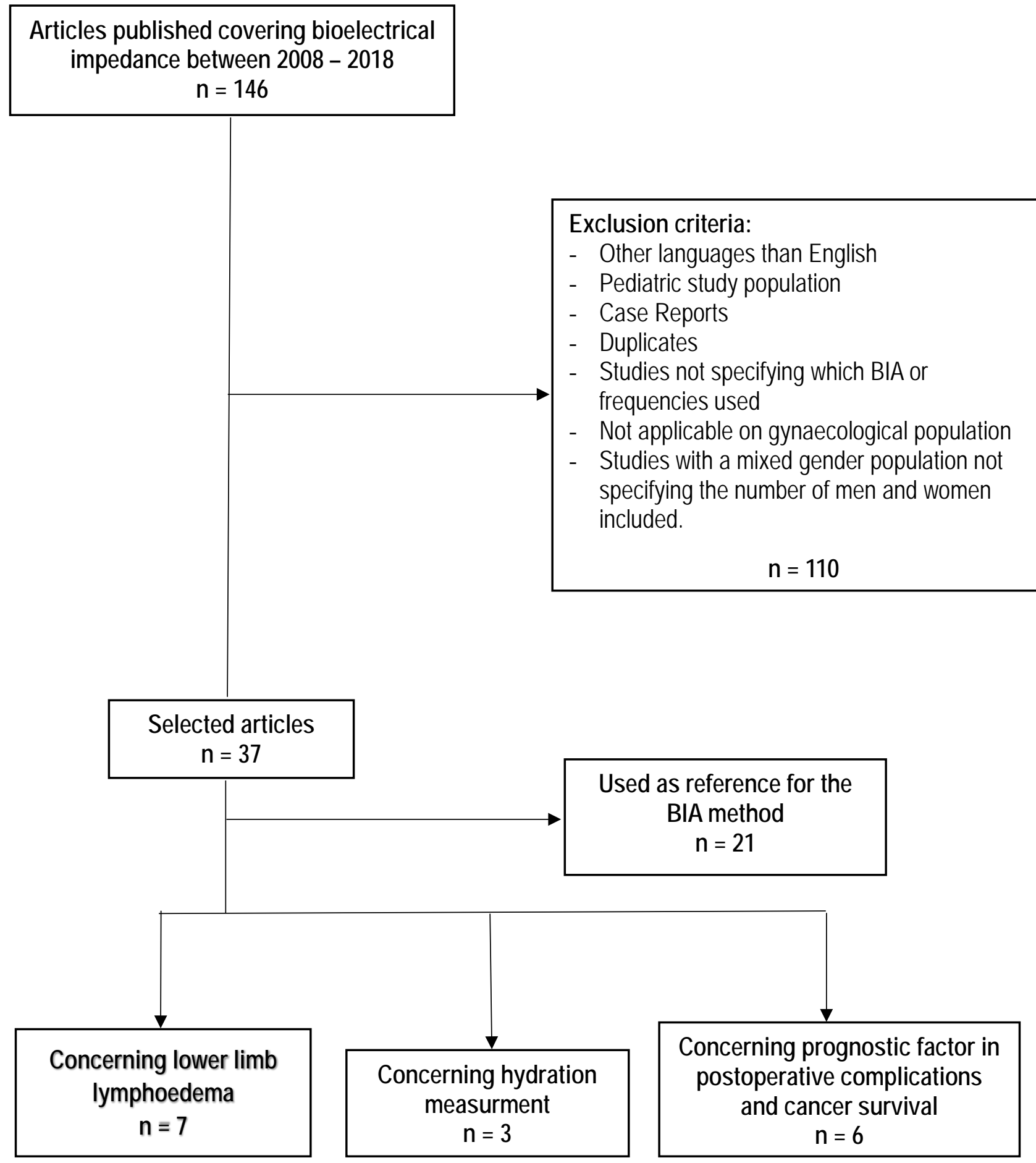

Flow chart 3. Selection of articles between 2008 - 2018, covering bioelectrical impedance analysis. 